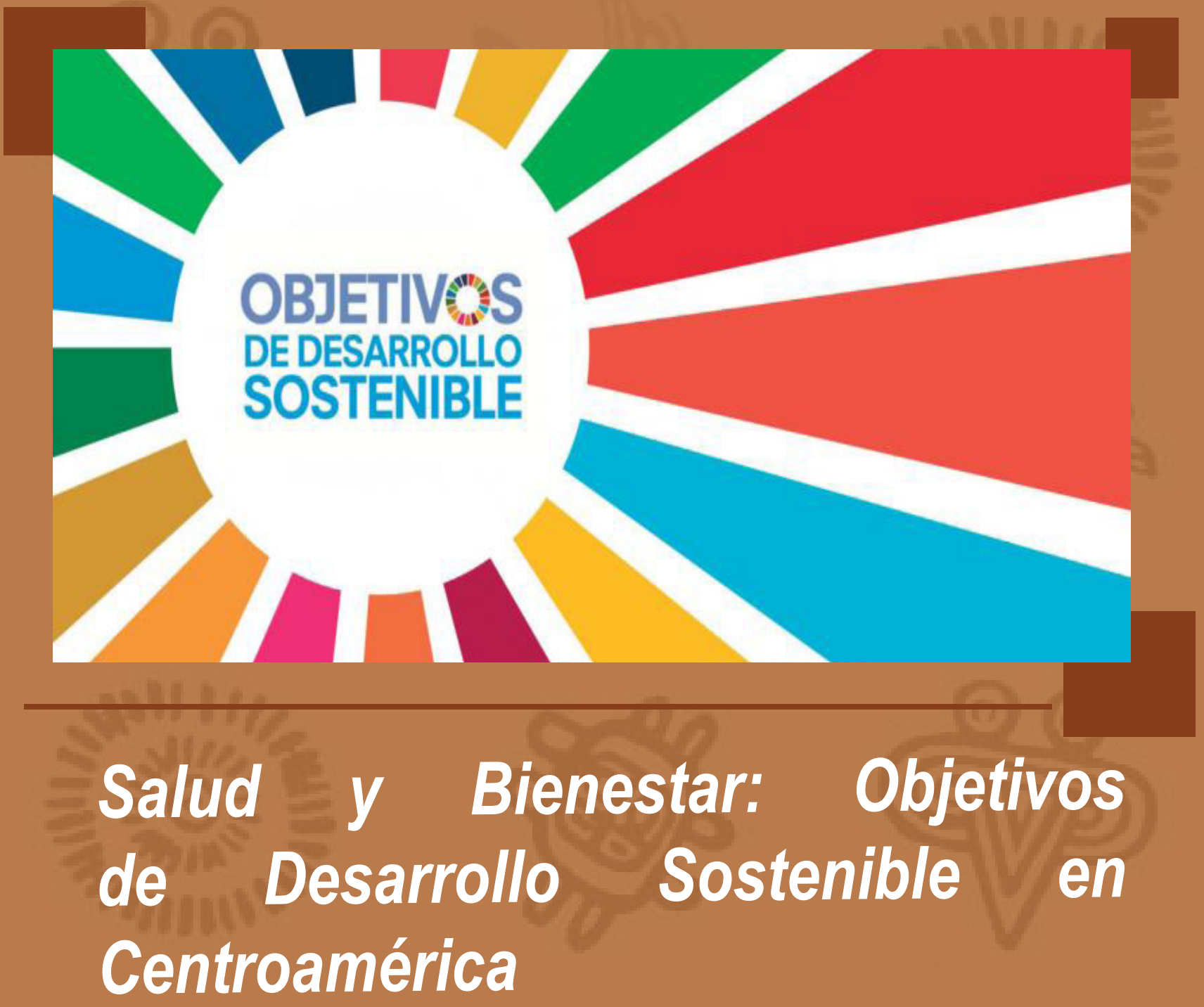

Estudios Interdisciplinarios 


\section{Salud y Bienestar: Objetivos de Desarrollo Sostenible en Centroamérica}

\section{Health and Wellbeing: Sustainable Development Goals in Central America}

Bryan Alexander Jaime Manzanarez

Docente-Investigador de la Universidad Nacional Autónoma de Nicaragua, Managua

(UNAN-Managua)

ID Orcid: https://orcid.org/0000-0002-7622-4962

bryan.jaime1609@gmail.com / bryan.jaime@unan.edu.ni
Recibido: 17-02-2021

Aceptado: 01-03-2021

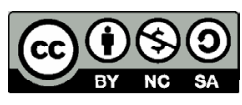

Copyright $\odot 2021$ UNAN-Managua Todos los Derechos Reservados.

\section{Resumen}

En este artículo científico se abordan los esfuerzos que realizan los Gobiernos de la Región Centroamericana en temas de Salud y Bienestar impulsados por las Naciones Unidas para dar salida y continuidad a la agenda de los Objetivos Desarrollo Sostenible (ODS) de los países en vías de desarrollo. De este enfoque, se examinarán las estrategias y los planes operativos que contrarresten la pandemia de la COVID-19 desde los sistemas de Salud públicos de los países centroamericanos, contribuyendo así al desarrollo social, económico, político y cultural de la Región Centroamericana. La salud pública y de calidad es un derecho que todo ser humano debe de poseer, sin ninguna exclusión como lo establecen los Derechos Humanos de las Naciones Unidas. La salud es el factor que más influye en el avance y progreso de las personas y las sociedades puesto que sin salud no hay vida. La salud además de asegurar una mejor calidad del entorno, también forma parte de los procesos cotidianos de nuestra vida, es por ello que la salud se encuentra garantizada en la mayoría de los países del mundo y su acceso ha sido considerado como un derecho humano esencial. Los Objetivos de Desarrollo Sostenible (ODS) destacan la importancia de garantizar y promover una salud adecuada, protegiendo el bienestar de toda la población. Para cumplir y dar respuesta a las necesidades es necesario impulsar iniciativas que erradiquen determinadas enfermedades, financiar los sistemas de salud, mejorar el saneamiento y aumentar el acceso a los servicios médicos. Es por eso, que los Gobiernos de Centroamérica realizan esfuerzos para cumplir con los ODS en el contexto de la COVID-19 y de esta forma garantizar el bienestar de las familias.

Palabras claves: Sistemas de Salud; Desarrollo Sostenible; Centroamérica; COVID-19, Derecho Humano.

\section{Abstract}

This scientific article addresses the efforts made by the Governments of the Central American Region on Health and Welfare issues promoted by the United Nations to give way out and continuity to the agenda of the Sustainable Development Goals (SDGs) of developing countries. From this approach, we will examine the strategies and operational plans that counteract the COVID-19 pandemic from the public Health systems of the Central American countries, thus contributing to the social, economic, political and cultural development of the Central American Region. Quality and public health is a right that every human being must possess, without any exclusion as established by the United Nations Human Rights. Health is the factor that most influences the advancement and progress of people and societies since without health there is no life. Health, in addition to ensuring a better quality of the environment, is also part of the daily processes of our life, that is why health is guaranteed in most countries of the world and access to it has been considered an essential human right. The Sustainable Development Goals (SDGs) highlight the importance of guaranteeing and promoting adequate health, protecting the well-being of the entire population. To meet and respond to needs, it is necessary to promote initiatives that eradicate certain diseases, finance health systems, improve sanitation and increase access to medical services. That is why Central American governments are making efforts to comply with the SDGs in the context of COVID-19 and thus guarantee the well-being of families. 


\section{Introducción}

La salud es el factor que más influye en el avance y progreso de las personas y las sociedades puesto que sin salud no hay vida. La salud además de asegurar una mejor calidad del entorno, también forma parte de los procesos cotidianos de nuestra vida, es por ello que la salud se encuentra garantizada en la mayoría de los países del mundo y su acceso ha sido considerado como un derecho humano esencial.

Existen organismos no gubernamentales e internaciones que velan por el cumplimiento del acceso a un servicio de salud gratuito y de calidad como derecho humano y en cumplimiento a los Objetivos de Desarrollo Sostenible, reivindicando las luchas de los pueblos en donde el acceso a la salud está privatizado producto de distintas causas, algunos de ellos son los procesos políticos, religiosos o económicos de cada región.

La Organización de las Naciones Unidas (ONU) considera que garantizar una vida sana y promover el bienestar de todos a todas las edades es importante para la construcción de sociedades prósperas:

El acceso a la salud y el bienestar es un derecho humano, y esta es la razón por la que la Agenda para el Desarrollo Sostenible ofrece una nueva oportunidad de garantizar que todas las personas, no solo las de mayor poder adquisitivo, puedan acceder a los más altos niveles de salud y asistencia sanitaria. (ONU, 2020)

La pandemia de la COVID-19 vino a cambiar el entorno de lo que se conocía como salud en los últimos años, muchos de los avances logrados por los países miembros de las Naciones Unidas han sufrido una contracción, debido a que toda la atención está puesta en la pronta recuperación de las personas y al desarrollo de una eventual vacuna para protegerse del Coronavirus.

En medio de la crisis sanitaria por la pandemia muchos de los países centroamericanos han implementado los mecanismos correctos y necesarios para desarrollar estrategias $y$ políticas públicas que aminore el gran impacto negativo de la pandemia durante su paso por la región.

En este artículo científico se argumentan los esfuerzos de los Gobiernos de Nicaragua, Guatemala y Honduras sobre las acciones que han desarrollado para asegurar la Salud y el Bienestar como Objetivo de Desarrollo Sostenible, y que, al mismo tiempo ha contribuido a disminuir significativamente los graves escenarios de COVID-19 que lamentablemente tienen otros países de la región centroamericana. 


\section{Metodología}

Según el alcance de esta investigación es de tipo descriptivo, puesto que interesa determinar los esfuerzos de los Gobiernos Centroamericanos para cumplir con los objetivos de Desarrollo Sostenible (ODS) y la Agenda 2030 en el contexto de la pandemia COVID-19. En cuanto a las características de los estudios de carácter descriptivo según Hernández, Fernández, Baptista afirman que "busca especificar propiedades, características y rasgos importantes de cualquier fenómeno que se analice, describe tendencias de un grupo de población" (2014, p.158). Además, la presente investigación utilizó como estrategia la investigación documental ya que según Fidias Arias (2006) plantea que la investigación se clasifica en: documental, de campo y experimental para responder al problema planteada.

La investigación tiene un enfoque cualitativo ya que se utilizó la recolección de información a través de la técnica de "observación" para obtener información, analizando las diversas notas informativas que difunden algunos medios centroamericanos. Además esta investigación tiene una perspectiva de estudio transversal ya que este estudio es donde se recolectan datos en un solo momento, en un tiempo único, su propósito es describir variables y su incidencia de interrelación en un momento dado, este de carácter no experimental (puesto que no se manipulan variables) ya que la investigación se enfocó en analizar y conocer los esfuerzos de los gobiernos centroamericanos para cumplir con los Objetivos de Desarrollo Sostenible en el contexto de la COVID-19.

Este estudio se realizó en los medios de comunicación latinoamericanos, específicamente abordando la temática de Salud en Centroamérica. Para esta investigación el universo fue conformado por diversos medios de comunicación escritos de formato digital a nivel latinoamericano. Así mismo, la muestra corresponde a 7 medios de comunicación digital debido a que estos medios abordan la temática de la salud y bienestar en los países centroamericano.

Para los métodos y técnicas de esta investigación se realizó un análisis del corpus de las siete notas informativas de los distintos medios de comunicación centroamericanos. La técnica que se utilizó para la recolección de información fue la observación de tipo "estructurada" ya que se utilizan instrumentos técnicos que guiaron y complementaron la investigación a través del análisis del corpus de noticias informativas.

La investigación a través del corpus de notas informativas cumple con los métodos de inclusión tales como:

Notas informativas actualizadas del año 2020

Medios de comunicación centroamericanos, pudiendo ser de Latinoamérica pero que hablen de Centroamérica.

La temática central sea dirigida al Objetivo de Desarrollo Sostenible (ODS) en el objetivo 3 (Salud y Bienestar). 
A partir de los datos recolectados en el proceso de investigación, se diseñaron bases para respaldar los datos obtenidos, utilizando el software Microsoft Word para Windows, prosiguiendo a la verificación de los datos recolectados y a los análisis de resultados.

En el proceso de la investigación se utilizaron diferentes tipos de herramientas para lograr una mejor eficacia, ejecución y procesamiento de la información. Todo esto para recoger datos de manera ordenada dentro del proceso de gestión de la información, tales como: Libreta, Lápiz, Teléfono inteligente, Computadora (con todos los programas de Microsoft Office)

\section{Discusión de Resultados}

Nicaragua: Su esfuerzo por la Salud y el Bienestar como Objetivo de Desarrollo Sostenible

Es un hecho que Nicaragua ya no es la misma desde hace muchos años, su transformación ha venido acompañada con motivos políticos, sociales o religiosos que han establecido la forma de gobernanza y sustentabilidad del país.

Uno de los grandes cambios del pueblo nicaragüense ha venido a impactar positivamente el acceso a la salud, pasando de una histórica privatización a un sistema que vela meramente por la gratuidad y el acceso igualitario a la misma como derecho humano, según declaraciones del secretario del Ministerio de Salud (MINSA), Dr. Carlos Sáenz en la revista matutina de Canal 4: Recordó que en el 2007 el buen Gobierno sandinista recibió del gobierno anterior un sistema de salud completamente deteriorado, donde prácticamente la mayoría de los servicios estaban privatizados. (Sáenz, 2020)

Esta investigación evidencia el total descuido del sistema de salud pública y la falta del presupuesto asignado por el gobierno de turno del entonces (2006), un gobierno que vino arrastrando la misma problemática durante 16 años debido a su costoso modelo capitalista aplicado al neoliberal: En el 2006 apenas se contaban con 34 máquinas de hemodiálisis y se atendía a 96 personas. Ahora cuentan con 332 y se atiende a más de 2 mil 200 personas para llevar mejor su vida y totalmente gratis. (Sáenz, 2020)

El Dr. Sáenz (2020) me nciona durante su intervención los procesos de cambios del modelo de salud familiar y comunitario que promueve el Gobierno de Reconciliación y Unidad Nacional desde que asumió el Poder en el año 2007: Desde 2007 hasta esta fecha se han puesto a funcionar 28 tomógrafos, 9 resonadores, antes los tomógrafos cobraban 300 dólares, desde 2007 se ha realizado 279 mil cirugías de ojos. (Sáenz, 2020)

Para el Dr. Saénz (2020) este modelo de salud no sólo se ha centrado en restituir los derechos de la salud para los niños, niñas, adolescentes, jóvenes y adultos, sino que también brinda acompañamiento especial a las mujeres embarazadas a través de 


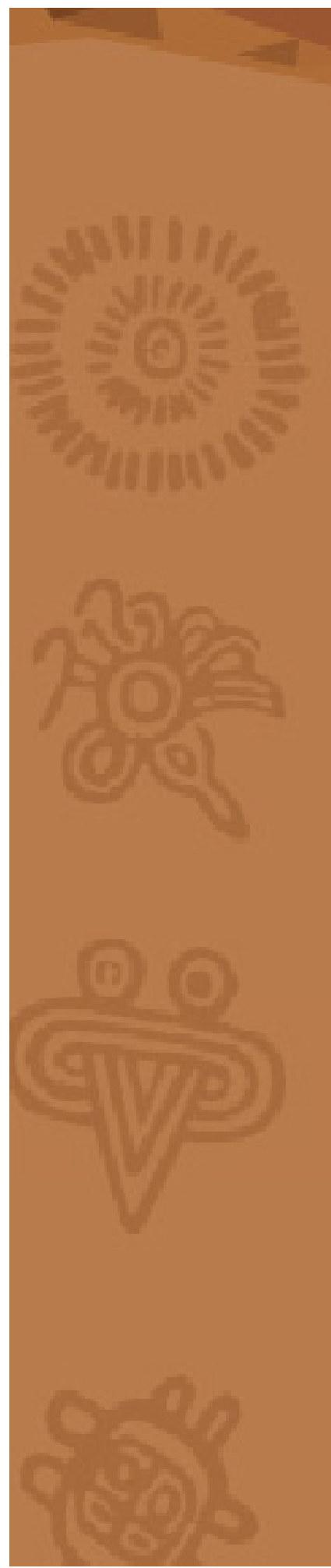

la atención gratuita en las casas maternas, que han contribuido a disminuir la tasa de mortalidad materna: En el 2009 se albergaron 66 mil personas en las casas maternas, que permite que antes de vayan a tener a sus niños, lleguen a un lugar más cercano de los centros de atenciones, lo que ha reducido la mortalidad materna en 66 por ciento desde el 2006. (Sáenz, 2020)

Gracias al compromiso que tiene el actual Gobierno de Nicaragua, dirigido por el Presidente Comandante Daniel Ortega, este país centroamericano ha sabido trabajar y contrarrestar la pandemia de la COVID-19, que, actualmente es uno de los países que no han detenido su economía ni sus avances pese a la crisis sanitaria internacional:

Se mantiene la lucha anti epidémica, anualmente se hacen 6 ciclos de aplicación de larvicida, visita casa a casa y fumigación domiciliar, además de charlas preventivas. Con toda esta disposición y voluntad política esto ayudó a poder contener de manera más rápida la situación del COVID y el Ministerio de Salud sigue en ese proceso.

\section{Gobierno Hondureño busca contrarrestar la Pandemia de la COVID-19}

Este país hondureño también ha demostrado avanzar en materia de salud como ODS para el pueblo, aunque con un sistema económico más deprimente y un gobierno menos preocupado por estas acciones. En los últimos meses han solicitado ayuda internacional para que les ayuden a contrarrestar los altos índices de contagiados por la COVID-19 logrando que unos "fondos de inversión" sean destinados para la compra de unidades móviles que atienda a contagiados por coronavirus.

Invest-H prometió entregar el hospital móvil en el transcurso de esta semana, sin embargo, este sábado aseguraron que personal de la Agencia de Regulación Sanitaria (ARSA) sigue con el proceso de revisión del equipo biomédico, por lo que se desconoce si la entrega podría tener más dilatorias.

Sin embargo, algunos de estos esfuerzos se han visto afectados por la lentitud de los procesos con el gobierno de turno, puesto que los medios de comunicación hablan de "seguimientos" casi todos los días, siendo que sea tan ansiosa la noticia de una inauguración:

Las pruebas y revisiones continuaron este sábado para poder entregarlos en las próximas horas a la Secretaría de Salud (SESAL)." "A un mes de haber comenzado su proceso de ubicación y equipamiento, Invest-H afirma que el avance en la instalación es de un 98 por ciento. (Pág.1)

Honduras no ha invertido solamente en estas móviles médicas para brindar atención a pacientes con COVID-19, sino que ha destinado una parte del presupuesto público para que tres especialistas de la Comisión de Salud viajen a Turquía para conocer técnicas que aporten a bajar los índices de contagios por el coronavirus. 
Gustavo Boquín (2020) interventor de Invest-H informó que el viaje a Turquía de los tres integrantes de la comisión cuesta poco menos de 200 mil lempiras. La población se mantiene expectante de recibir noticias sobre la comisión que partió de Honduras con rumbo a Turquía para constatar el proceso de los cinco hospitales móviles restantes. (Boquín, 2020)

Pese a estas dificultades, Honduras apuesta por el funcionamiento de estos hospitales móviles y han incluido una tecnología específica para su funcionamiento. Según los especialistas, estas móviles iniciarían a funcionar en el mes de octubre:

Algunas de las tecnologías que tendrán los hospitales móviles que llegaron el país el pasado mes de julio, sin embargo, para iniciar a funcionar faltan otros equipos y estos estarían llegando al país a finales del mes de septiembre y comenzarían a operar en octubre en las diferentes ciudades. (Boquín, 2020)

\section{Presidente de Guatemala inaugura cinco hospitales tempo- rales en un año}

Es muy interesante comprender como influyen las formas de concentración del Poder entre los Gobiernos del mundo, específicamente en el caso de Guatemala, éste había tenido una forma de gobierno bastante particular en los últimos 40 años con varios déficits en salud y educación acompañado de un costosísimo modelo neoliberal.

Hoy la realidad de este país centroamericano es otra, con un gobierno trabajando para la satisfacción del pueblo, una "revolución" en el gobierno guatemalteco dirigido por el presidente Alejandro Giammatei que tomó posesión en enero de este año, bajo un partido político de derecha "nuevo y diferente".

Una de sus destacas obras que benefician al pueblo de Guatemala y ratifican su compromiso con los Objetivos de Desarrollo Sostenible (ODS), tenemos las inauguraciones de los hospitales que atienden a pacientes contagiados por la COVID-19 y su visión hacia el futuro: Alejandro Giammattei, inauguró hoy el quinto hospital temporal para atender a pacientes Covid-19...porque ahora el gran reto que tenemos para el año entrante es construir siete centros más en Guatemala. (El País, 2020)

Guatemala apuesta a una transformación en el antes debilitado sistema de salud, en donde el presidente ha ratificado el aumento del presupuesto destinado para esta área y además resalta sus planes para el futuro, suponiendo que al presidente le restan tres años más en el Poder:

Este no es el final de una etapa, es a duras penas el comienzo, porque ahora el gran reto que tenemos para el año entrante es construir siete centros más en Guatemala. Giammattei aseguró que el presupuesto para 2021 tiene que enfocarse en dos acciones, la primera, la salud pública abandonada por 40 años. (El País, 2020) 


\section{¿Qué hacen los Gobiernos de la Región Centroamericana para combatir la Pandemia de la COVID-19? Una preocupación mun- dial.}

Como parte del estudio realizado se aplicó la técnica de recolección de datos (observación de tipo estructurada) del corpus de siete notas informativas de distintos medios de comunicación centroamericanos, para analizar los esfuerzos que realizan los gobiernos de la región en temas de Salud y Bienestar impulsados por las Naciones Unidas para dar salida y continuidad a la agenda de Desarrollo Sostenible (ODS) de los países en vías de desarrollo, de esta forma esta técnica se implementó con el objetivo de obtener información en temas de salud y bienestar, desde las diferentes editoriales (Medios de Comunicación Masivos) de los países de Centroamericanos.

\section{Gobierno de Nicaragua desarrolla y mejora planes estratégicos en el Sistema de Salud Nacional para combatir y contrarrestar la Pandemia de la COVID-19.}

Una nueva historia se vive en Nicaragua, después de muchos años de privatización de derechos e inclusión social ante los gobiernos neoliberales el país se convierte en uno de los principales países en vías de desarrollo a nivel centroamericano, al alcanzar un Sistema de Salud público eficiente y de calidad, contrarrestando la pandemia de la COVID-19 a través de los Objetivos de Desarrollo Sostenible (ODS), planificación, creación de políticas públicas ante amenazas, riesgos de enfermedades y problemas sociales que se presenten en el país.

El Diario Digital refleja los resultados y avances en materia de salud pública y de calidad que el Frente Sandinista de Liberación Nacional (FSLN) ha impulsado desde que regresó al Gobierno hace más de 13 años. Destacando que en Nicaragua existen 143 centros de salud, mil 333 puestos médicos, 178 casas maternas y clínicas móviles que ayudaran a contrarrestar la pandemia mundial de la COVID-19, resaltando que el primer decreto del presidente, Daniel Ortega, el 11 de enero del 2007 fue dejar sin efecto la llamada autonomía escolar, restituyó el derecho constitucional a la salud y su acceso gratuito y universal.

Por otro lado, en avances de salud pública y mejoramiento de los sistemas de salud y cumplimiento de los (ODS) se resaltó que en Nicaragua desde el 2007 se han construido 18 hospitales nuevos y actualmente hay seis más en ejecución y que toda la atención médica está dirigida a la comunidad en este contexto de salud pública, como es el de la pandemia de la COVID-19. Por lo que es necesario fortalecer los sistemas de salud públicos del país, en materia de atención pública y de calidad. Puesto que desde 2006 se mejora el acceso a la salud de un 34.1 al 92.8 por ciento en la actualidad, la tasa de mortalidad infantil pasó del 29 al 12 por ciento, según datos del Ministerio de Salud (MINSA, 2020).

Con estas características más sobresalientes en temas de salud pública y bienestar ciudadana, el Gobierno de Nicaragua del Frente Sandinista de Liberación Nacional (FSLN) ha impulsado planes y estrategias para combatir las debilidades y amenazas 
que puede presentar el sistema de salud del país, previo a las amenazas, brotes y coyuntura mundial de la pandemia de la COVID-19, puesto que solo una verdadera revolución asume las obras en materia de salud que se han impulsado a lo largo de los años a través de la restitución de derechos a las familias pobres, inclusión de los históricamente excluidos, capitalización de las familias pobres, inversión en bienes públicos que benefician a todos, creando paz y seguridad y un crecimiento con menos pobreza y más igualdad.

En este proceso de amenaza que tienen los países centroamericanos ante la pandemia de la COVID-19, el país de Nicaragua a través del modelo socialista que implementa el Gobierno del Frente Sandinista se destaca por la creación de planes estratégicos que ayudan al desarrollo sostenible del país, combatiendo y contrarrestando las amenaza que se pueden presentar en el momento longitudinal de la pandemia de la COVID-19.

Es importante mencionar que los cambios y el desarrollo sostenible de los países centroamericanos, van vinculados a la gestiones y planificaciones de los gobiernos actuales, es por ello que en Nicaragua sobresalen las buenas funciones que desempeña el gobierno del Frente Sandinista de Liberación Nacional, puesto que desde los primeros casos de habitantes que dieron positivo a la COVID-19 se crearon mecanismos de prevención y estrategias para evitar un brote y saturación de los Sistema de Salud Públicos del país.

\section{¿Hospitales Colapsados o Sistema de Salud deficientes en el Salvador? Preocupación regional.}

Antes las debidas amenazas que se presentan a lo largo del contexto coyuntural ante la pandemia de la COVID-19, el Sistema Salud público del país de El Salvador sufre un colapso tras recibir un brote grado tres de corona virus, ya que según diarios oficiales del país, aseveran que: médicos afirma que los hospitales del país han colapsado y que no cuentan con recursos médicos, y que los planes de desarrollo y de mejora continua a los servicios de salud pública con los que no cuenta el país no es el mejor.

En este sentido, el país de El Salvador no cuenta con las medidas y recursos necesarios para afrontar amenazas y riesgos que están presente ante la enfermedad de la COVID-19 en los países centroamericanos, pues que según los datos del Diario Hoy los profesionales de la salud, quienes han atendido un total de 4,475 casos positivos a la COVID-19, también luchan contra el cansancio y la falta de recursos médicos para atender a los enfermos, y cada día llegan más y más, cobrando la vida de 93 pacientes en nuestro país.

Ante la falta de cumplimiento de los Objetivos de Desarrollo Sostenible (ODS) para los países de en vías de desarrollo, como es el caso del país de El Salvador que cuenta con una economía en crecimiento al no ser un país agrícola, pero si industrial, este carece de una planificación estructurada que brinde soluciones a las amenazas que se presente durante la pandemia de la COVID-19. 


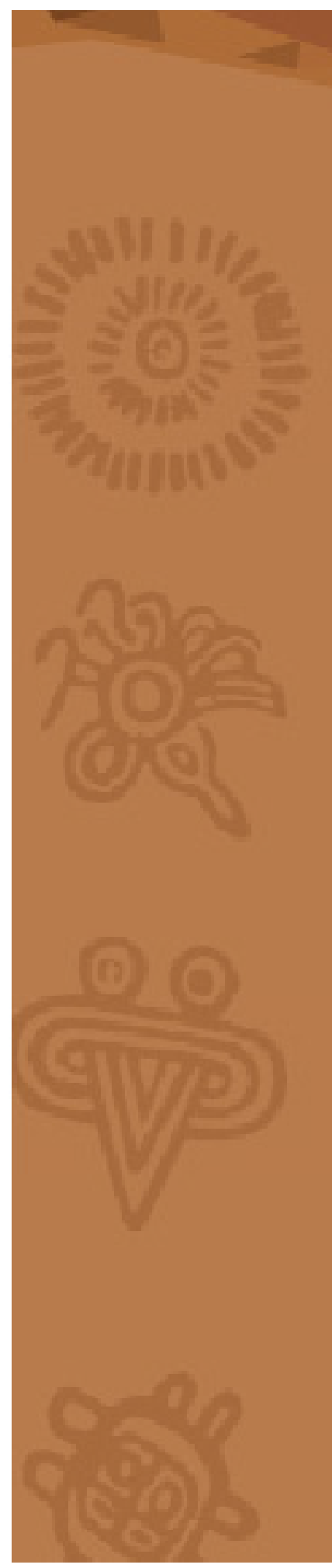

De esta forma no resulta extraño que, ante este escenario, los galenos no dudan en afirmar que el sistema de salud ya colapsó, con hospitales saturados, sin el equipo médico necesario para la atención, incluso sin oxígeno para aquellos casos más graves, aseveran datos cualitativos del Diario Digital "El Hoy".

En efecto, ante el contexto coyuntural de la pandemia de la COVID-19 se considera por parte del Ministerio de Salud que el aumento del número de pacientes positivos no se debe a la apertura económica que arrancó el pasado 16 de junio. Ese efecto, se experimentará en unos 15 días con un rebrote de casos.

Dicho en otros términos, según datos de los profesional de la salud que trabaja en el Hospital General del ISSS Central de El Salvador, que este efecto se debe a que los hospitales carecen de recursos médicos y planes operativos para contrarrestar el colapso de los centros hospitalarios de El Salvador, tomando en cuenta que hay pacientes que se están muriendo en los pasillos, porque llegan bien delicados y la falta de oxígeno o espacios equipados para este tipo de atención no permite al personal de salud atenderlos adecuadamente. En otros términos, el país de el Salvador debe de crear políticas públicas que contribuyan al desarrollo de los sistemas de salud públicos, que brinden a la comunidad salud pública y de calidad, en caminado al objeto número tres de los Objetivos de Desarrollo Sostenible (ODS) a través del Programa de las Naciones Unidad para el Desarrollo (PNUD) en América Latina y el Caribe.

Al no poseer planes estratégicos y políticas públicas que contribuyan al desarrollo del país, los sistemas de salud de El Salvador no darán abasto tras el contexto de la pandemia COVID-19 en grado tres de transmisión infecciosa. Puesto que el Dr. Juan José Cálix, del Hospital San Rafael. Refiere que se sienten impotentes al querer ayudar y sentir que es imposible hacerlo, por más que luchemos. El sistema ya no da abasto.

Al poseer complicaciones directas el Gobierno actual de El Salvador anuncia: La apertura de la primera fase del hospital del Centro Internacional de Ferias y Convenciones (CIFCO), que será inaugurado este domingo, contribuirá a disminuir la saturación existente, evitando el contagio masivo y desaturando el Sistema Nacional de Salud. Puesto que se espera que el país avance en materia de salud pública y de calidad para contribuir al desarrollo y mejoramiento político, social y económico en pro a su desarrollo integral como país en busca de desarrollo, como lo establecen los Objetivos de Desarrollo Sostenibles (ODS).

\section{EI Salvador apuesta por descubrir vacuna para la COVID-19}

Uno de los medios de comunicación llamado La Grafica periódico digital matutina, ubicado en El Salvador describe el avance que se realiza sobre la vacuna del Covid-19 con que se pretende disminuir los contagios de esta pandemia. Esta vacuna se busca se busca por dos vías: una con la gestión que hace la Organización Panamericana de la Salud (OPS) y la segunda directamente con los laboratorios que están en la fabricación de la inmunización para que sea accesible para toda la población salvadoreña. 


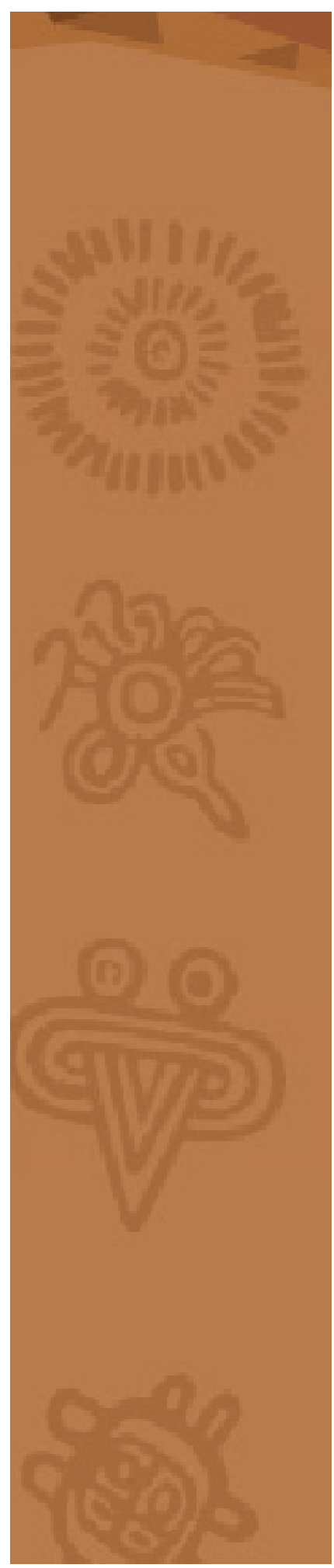

El ministro dijo que desde el inicio de la pandemia se nombró un equipo para que se le diera seguimiento al desarrollo a los protocolos y avances, este mismo equipo ha seguido los diferentes tratamientos médicos que se han aplicado a los pacientes para su recuperación. Además, insta a toda la población a que tomen las medidas de seguridad e higiene para evitar contraer la enfermedad.

\section{Nicaragua, el ejemplo del modelo de salud familiar y comuni- tario pre-pandemia}

Por otro lado, el canal de televisión venezolano TeleSur a través de una fuente de Prensa Latina: El 19 Digital, pretende comunicar a la población latinoamericana acerca de los avances que existen desde el año 2018 a 2019 en Nicaragua sobre la reducción del cáncer en las mujeres. El Gobierno de Nicaragua pretende ayudar y dar respuesta a las necesidades de la población por medio de salud gratuita y de calidez a todas aquellas que lo necesitan.

La nota refiere al avance que se realiza en Nicaragua para dar respuesta a las necesidades de las mujeres con cáncer. El gobierno ha puesto interés en reducir el riesgo de muerte por esta enfermedad; brindando equipos de alta gama y una atención de calidad hacia la población.

Actualmente, el mundo se enfrenta a una crisis sanitaria mundial sin precedentes; la COVID-19 está propagando el sufrimiento humano, desestabilizando la economía mundial y cambiando drásticamente las vidas de miles de millones de personas en todo el mundo.

Antes de la pandemia, se consiguieron grandes avances en la mejora de la salud de millones de personas. En concreto, estos grandes avances se alcanzaron al aumentar la esperanza de vida y reducir algunas de las causas de muerte comunes asociadas con la mortalidad infantil y materna. Sin embargo, se necesitan más esfuerzos para erradicar por completo una gran variedad de enfermedades y abordar un gran número de problemas de salud, tanto constantes como emergentes. A través de una financiación más eficiente de los sistemas sanitarios, un mayor saneamiento e higiene, y un mayor acceso al personal médico, se podrán conseguir avances significativos a la hora de ayudar a salvar las vidas de millones de personas.

Las emergencias sanitarias, como la derivada de la COVID-19, suponen un riesgo mundial y han demostrado que la preparación es vital. El Programa de las Naciones Unidas para el Desarrollo señaló las grandes diferencias relativas a las capacidades de los países para lidiar con la crisis de la COVID-19 y recuperarse de ella. La pandemia constituye un punto de inflexión en lo referente a la preparación para las emergencias sanitarias y la inversión en servicios públicos vitales del siglo XXI. 


\section{Conclusión}

La Región Centroamericana ha tenido un cambio y un avance significativo en temas de salud pública y de calidad en los últimos tiempos, puesto que algunos sistemas políticos de los países centroamericanos han ejecutado planes operativos por medio de los Objetivos de Desarrollo Sostenible (ODS) impulsados por las Naciones Unidas para combatir la pandemia de la COVID-19, a través del objetivo número tres de Salud y Bienestar que contribuye al desarrollo sostenible de los sistema de salud públicos de los países en vías de desarrollo.

De esta forma, países de la Región Centroamérica avanzan en materia de salud pública y de calidad, tal es el caso de los países de Nicaragua y Guatemala que están ejecutando planes y estrategias operativas a través de los Objetivos de Desarrollo Sostenible (ODS), promoviendo una inclusión social para el desarrollo de sus naciones. Sin embargo, el Gobierno de Nicaragua destaca su compromiso con el pueblo nicaragüense ya que su buena gestión se evidenció en el contexto de la COVID-19 donde el país obtuvo mejores respuestas para evitar la propagación infecciosa y exponer a los ciudadanos a contraerlas.

La pandemia es mucho más que una crisis sanitaria, requiere de una respuesta de los Gobiernos y la sociedad en su conjunto equivalente a la determinación y el sacrificio de los trabajadores sanitarios en primera línea. Es por eso, que todos los gobiernos centroamericanos buscan dar soluciones y respuestas inmediatas a la población necesitada.

Sin embargo, países de la región deben de trabajar en creación de políticas públicas en caminadas al cumplimiento de los Objetivos de Desarrollo Sostenible (ODS) que contribuya a que sus países traten de evitar y contrarrestar riesgos y amenazas que se presenten a lo largo de los años en temas de Salud y bienestar.

\section{Lista de Referencias}

El 19 Digital. (2020). ¿Cuáles son los avances en materia de salud que ayudaron a enfrentar la pandemia en Nicaragua? Extraído de: https://www.el19digital.com/articulos/ver/titulo:105986cuales-son-los-avances-en- materia-de-salud-que-ayudaron-a-enfrentar-la-pandemia-en-nicaragua

El Heraldo. (2020). Instalación de hospitales móviles en Honduras avanza en un 98 por ciento. Consultado en: https://www.google.com/amp/s/www.elheraldo.hn/pais/1404823-466/hospitalmoviles-honduras-investh $\% 3$ famp $=1$

El País. (2020). Presidente guatemalteco apuesta por mejorar salud pública en 2021. Recuperado de: http://www.elpais.cr/2020/09/02/presidente-guatemalteco-apuesta- por-mejorar-salud-publica-en-2021/ 


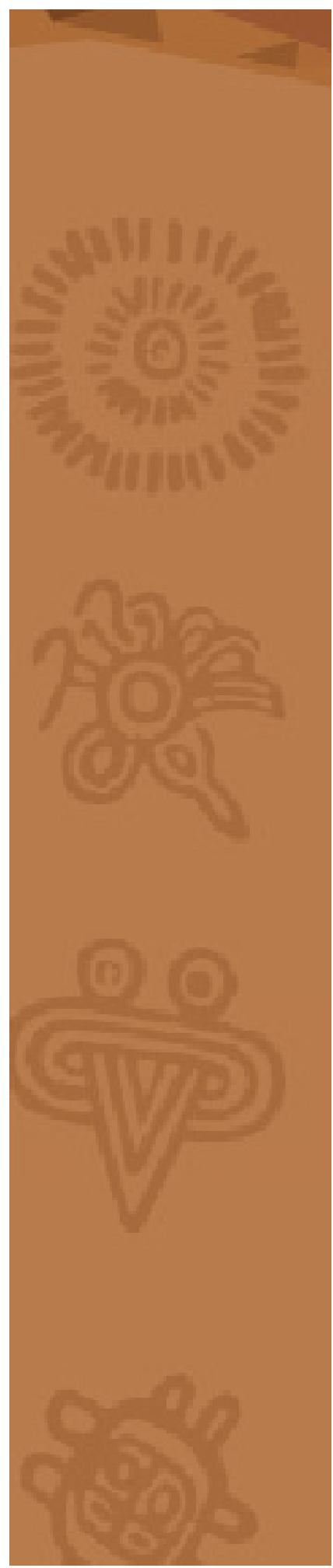

ElSalvador.com. (2020). Médicos confirman que hospitales de El Salvador han colapsado. Extraído de: https://www.elsalvador.com/eldiariodehoy/hospitales-colapsados- pacientes-covid19-el-salvador/725796/2020/

Jaime, B. (2020). Políticas Públicas en la Educación Superior Virtual Nicaragüense. Raíces: Revista Nicaragüense de Antropología. Consultado en: https://doi.org/10.5377/raices.v3i7.9697

La Prensa Gráfica. (2020). Ministerio de Salud busca vacuna de covid-19 por dos vías. Consultado en: https://www.laprensagrafica.com/elsalvador/Ministerio-de-Salud- busca-vacuna-de-covid-19por-dos-vias-20200820-0094.html

Organización de las Naciones Unidas. (2020). Salud y Bienestar: Por qué es importante. Extraído de: https://www.un.org/sustainabledevelopment/es/wp-content/uploads/sites/3/2016/10/3_Spanish_Why_it_Matters.pdf

Prensa Latina. (2020). Destacan avances en salud de Nicaragua durante el gobierno del FSLN. Recuperado de: https://www.elpais.cr/2020/07/16/destacan-avances-en- salud-de-nicaragua-durante-el-gobierno-del-fsin/

Telesur. (2020). Nicaragua destaca avances en la lucha contra el cáncer. Extraído de: https:// www.google.com/amp/s/www.telesurtv.net/amp/news/nicaragua-destacaavances-lucha-cancer-20200205-0034.html

Lic. Bryan Alexander Jaime Manzanarez

Docente-Investigador de la Universidad Nacional Autónoma de Nicaragua, Managua (UNAN-Managua), Carrera de Comunicación para el Desarrollo de la Facultad de Humanidades y Ciencias Jurídicas. Coordinador del Sistema de Comunicación (SISCOM) de la Facultad de Humanidades y Ciencias Jurídicas. Ha elaborado ensayos e investigaciones en el marco de la aplicación de Plan de Comunicación para proyectos institucionales (2020). Políticas Públicas en materia de salud y educación (2018). Elaboración de un Proyecto de Comunicación donde se proponía la creación una institución educativa para niños y jóvenes en Carazo, de acuerdo a uno de los ODS de la CEPAL (2018). Elaboración de un reportaje radial sobre la Inversión Pública en Educación Superior Virtual (2018). Elaboración de campañas publicitarias en la prevención de embarazos a temprana edad en Jinotega. (2018). Participación en Ponencias sobre Integración Centroamericana impartidas por el Parlamento Centroamericano (PARLACEN) y la Corte Centroamericana de Justicia 